\title{
SÉRAPHIN, G. (dir.) - Religion, guérison et forces occultes en Afrique. Le regard du jésuite Éric de Rosny
}

Valérie Nne'e Onna

\section{OpenEdition}

\section{Journals}

Édition électronique

URL : http://journals.openedition.org/etudesafricaines/20650

DOI : 10.4000/etudesafricaines.20650

ISSN : 1777-5353

Éditeur

Éditions de l'EHESS

\section{Édition imprimée}

Date de publication : 1 avril 2017

Pagination : 204-207

ISBN : 978-2-7132-2688-5

ISSN : 0008-0055

Référence électronique

Valérie Nne'e Onna, "SÉRAPHIN, G. (dir.) - Religion, guérison et forces occultes en Afrique. Le regard du jésuite Éric de Rosny », Cahiers d'études africaines [En ligne], 225 | 2017, mis en ligne le 01 avril 2017, consulté le 24 septembre 2020. URL : http://journals.openedition.org/etudesafricaines/20650 ; DOI : https://doi.org/10.4000/etudesafricaines.20650

Ce document a été généré automatiquement le 24 septembre 2020.

(c) Cahiers d'Études africaines 


\title{
SÉRAPHIN, G. (dir.) - Religion, guérison et forces occultes en Afrique. Le regard du jésuite Éric de Rosny
}

\author{
Valérie Nne'e Onna
}

\section{RÉFÉRENCE}

SÉRAPHIN, G. (dir.) - Religion, guérison et forces occultes en Afrique. Le regard du jésuite Éric de Rosny. Paris-Yaoundé, Karthala-UCAC, 2016, 264 p., bibl., ill.

1 Éric de Rosny, jésuite, initié au ndimsi (le «monde de la nuit » chez les Douala) et ethnologue, disparaissait le 2 mars 2012, laissant derrière lui une œuvre remarquable, produit de plusieurs décennies d'actions et de recherches principalement au Cameroun. Pour lui rendre hommage, un colloque international intitulé « Le pluralisme (médical, religieux, anthropologique, juridique...) en Afrique. Le regard d'Éric de Rosny » a été organisé à l'Université catholique d'Afrique centrale les 9 et 10 décembre 2014. Cet ouvrage prolonge cette rencontre sans en être les actes, comme le précise Gilles Séraphin qui en assure la direction scientifique.

2 Comme le colloque, le livre souligne la contribution riche et plurielle d'Éric de Rosny au champ de la connaissance. Il réunit dix-huit textes signés par une vingtaine d'auteurs aux profils extrêmement divers (sans surprise de nombreux ethnologues et anthropologues, mais aussi des médecins, des psychologues, des politologues ou encore des historiens). L'ouvrage est introduit par un double hommage avec une préface d'Ekwalla Malobe, représentant du monde des nganga (guérisseurs traditionnels) et le propos liminaire de Gilles Séraphin. Le premier revient sur les circonstances de sa rencontre avec le père de Rosny et sur le cheminement de ce dernier dans la communauté douala, particulièrement auprès des guérisseurs traditionnels. Il insiste 
tout spécialement sur l'activisme d'Éric de Rosny pour la sauvegarde de la médecine traditionnelle et la réhabilitation du travail de ces thérapeutes aujourd'hui confondus aux sorciers, leurs ennemis mortels. Dans son introduction, Gilles Séraphin revient sur l'organisation du colloque de 2014 destiné à éclairer la singularité du travail d'Éric de Rosny et à souligner sa postérité scientifique. Celle-ci est avant tout une "matière " scientifique contenue dans ses travaux, menés principalement sur «le monde de la nuit », mais aussi sur des thématiques connexes telles que la pharmacopée, le droit ou le développement de nouvelles pratiques religieuses. L'intérêt de son œuvre pour la science est également relatif à sa méthode et à son positionnement extrêmement particuliers, ce que Gilles Séraphin nomme le « regard situant».

Dans la première partie de l'ouvrage, les articles de Thomas Théophile Nug Bissohong (chargé de cours à l'Université de Douala), Roberto Beneduce (anthropologue et psychiatre), Jacques Fédry (linguiste jésuite) et André Mary (anthropologue) mettent en parallèle le parcours personnel et l'évolution scientifique d'Éric de Rosny qui était à la fois prêtre, « initié » et ethnologue. La conjonction de ces statuts a priori antinomiques n'était pas exempte d'une certaine ambiguïté qui, pour André Mary, était nécessaire au maintien de cet équilibre. Le refus des catégories limitatives évoque la quête effrénée de liberté qui l'avait déjà poussé vers l'engagement missionnaire, puis à la découverte des maîtres de la nuit au Cameroun. Si Éric de Rosny n'est pas le premier prêtreethnologue, ni le premier ethnologue-«initié », son parcours se singularise par la capacité qu'il a eue d'occuper chacun de ces terrains sans se perdre de vue, à aller vers l'autre sans vouloir se substituer à lui. S'il ne s'identifiait pas comme nganga en dépit de son initiation ou comme ethnologue malgré ses recherches, il n'a jamais remis en cause son statut d'ecclésiastique. C'est ce profond attachement aux convictions de sa mission évangélique qui, d'après ces auteurs, lui a permis d'assumer pleinement ces différents rôles et de livrer des commentaires inédits. Nug Bissohong souligne comment cette « littérature du moi » (son œuvre étant grandement autobiographique) s'est structurée autour de moments-clés de son parcours personnel. Son œuvre s'est construite dans un mouvement itératif permanent entre soi et l'«autre» avec pour préoccupation perpétuelle la recherche de ce qui trouble le bien-être de l'individu et des moyens de le " guérir » en soignant ses angoisses existentielles in situ. Les différents auteurs insistent également sur l'influence d'Ignace de Loyola (fondateur de la Compagnie de Jésus à laquelle il appartenait) sur le travail d'Éric de Rosny. Son initiation ignatienne l'aurait indubitablement préparé à saisir le sens des thérapies des nganga et à recevoir sa seconde initiation, la réception de la «double vue ». Cette première partie se conclut sur deux nouveaux hommages: celui de Patrice Mbaya, membre du Groupe de recherche sur la sorcellerie (GRS) fondé par Éric de Rosny et celui d'Anne-Nelly PerretClermont, professeur honoraire de l'Université de Neuchâtel au sein de laquelle Éric de Rosny a reçu un doctorat honoris causa en 2010.

4 Dans la deuxième partie de l'ouvrage, Jean-François Bayart (politologue), Peter Geschiere (anthropologue) et Alain Henry (ingénieur et sociologue) reviennent sur la manière dont les années de collaboration et souvent d'amitié avec Éric de Rosny ont influencé leurs itinéraires de chercheurs. Ainsi apprend-on que l'approche du "politique par le bas » développée par Jean-François Bayart n'aurait pas pris corps, tout au moins sous sa forme actuelle, sans l'acuité des échanges avec Éric de Rosny. Bayart note à ce sujet : «si j'ai apporté quelque chose de neuf à la compréhension du politique en Afrique, au fil de ma carrière, c'est en grande partie grâce à ce dialogue » 
(p. 76). De même, Alain Henry indique comment le regard d'Éric de Rosny lui a ouvert de nouvelles perspectives dans l'analyse de la gestion entrepreneuriale au Cameroun.

5 Les nombreux articles réunis dans la troisième partie du livre attestent d'une des particularités de l'œuvre d'Éric de Rosny, à savoir sa capacité à transcender les clivages qu'ils soient disciplinaires ou épistémologiques. Les neuf articles de la troisième partie montrent comment les travaux récents prolongent ou élargissent le champ des réflexions développées par de Rosny dans les domaines du religieux (pour les textes d'Yvan Droz sur le développement du concept de « Butinage religieux » sous l'influence d'Éric de Rosny, de Berthe Lolo, Andrea Ceriana Mayneri et d'Edmond Mballa Elanga), de la santé (textes de Sandra Fancello, Marie-Thérèse Mengue et Fatimatou Mounsadé Kpoundia) ou du management des entreprises (Emmanuel Kamdem et Henri Tedongmo Teko). Mathieu Lavoyer et Jean-Daniel Moredod évoquent quant à eux les apports des analyses rosniennes à la compréhension d'aspects de la sorcellerie médiévale européenne ignorés par le modèle savant. Outre soutenir des réflexions novatrices sur les formes d'expression de l'invisible dans les sociétés humaines anciennes et contemporaines, d'inspirer de nouvelles formes d'engagement sur le terrain, l'œuvre et la biographie d'Éric de Rosny peuvent également être de passionnants objets d'étude comme l'illustre l'analyse d'Henri Njengoué Ngamaleu sur la construction et les enjeux de l'interculturalité.

6 L'ouvrage se conclut presque comme il a commencé sur une postface de Dinh Boniface Alfred Mandengue, co-président de l'« Association des amis d'Éric de Rosny », suivie de la bibliographie complète d'Éric de Rosny établie par Anne-Nelly Pierre-Clermont.

7 La diversité de ces contributions - parfois de qualité inégale - inspirées par le regard ou les analyses d'Éric de Rosny témoigne de la variété des usages qui peuvent être appliqués à son œuvre. Ces analyses et témoignages rendent compte de l'importance et de l'actualité des apports d'Éric de Rosny aux domaines de la connaissance. Outre l'hommage à celui qui fut un ami, un collègue ou un guide pour la plupart des auteurs, cet ouvrage permet de faire la lumière sur une œuvre encore trop confidentielle (sans doute a-t-elle hérité de la discrétion de son auteur) en dépit de sa richesse et de son influence indéniable sur l'analyse $d u$ fait religieux et sorcier dans l'Afrique contemporaine. En plus de son œuvre, c'est une conception inédite du métier d'ethnologue qu'Éric de Rosny lègue aux nouvelles générations de chercheurs. Cet ouvrage a l'infini mérite d'en rendre compte en plus d'ouvrir de nouvelles perspectives de recherches extrêmement intéressantes en Afrique et ailleurs. Autant d'éléments qui en font un ouvrage d'une importance certaine. 